\title{
Exclusive production of dijets in QCD
}

\author{
D.Yu. Ivanov a b \\ anstitut für Theoretische Physik, Universität Regensburg, D-93040 Regensburg, Germany \\ ${ }^{\mathrm{b}}$ Institute of Mathematics, 630090 Novosibirsk, Russia
}

We study in a framework of QCD collinear factorization the processes of a pion and a photon diffraction dissociation into two jets. The structure of non-factorizable contributions is discussed. We argue that coherent production of hard dijets by linearly polarized real photons can provide direct evidence for chirality violation in hard processes, the first measurement of the magnetic susceptibility of the quark condensate and the photon distribution amplitude.

\section{Introduction}

The pion (and photon) diffraction dissociation into a pair of jets with large transverse momentum on a nucleon target was first discussed in [1. The possibility to use this process to probe the nuclear filtering of pion components with a small transverse size was suggested in [2]. The A-dependence and the $q_{\perp}^{2}$-dependence of the coherent dijet cross section was first calculated in [3] and it was argued that the jet distribution with respect to the longitudinal momentum fraction has to follow the quark momentum distribution in the pion and hence provides a direct measurement of the pion distribution amplitude. Recent experimental data by the E791 collaboration [ 1 indeed confirm the strong A-dependence which is a signature for color transparency, and are consistent with the predicted $\sim 1 / q_{\perp}^{8}$ dependence on the jet transverse momentum. Moreover, the jet longitudinal momentum fraction distribution turns out to be consistent with the $\sim z^{2}(1-z)^{2}$ shape corresponding to the asymptotic pion distribution amplitude. After these first successes, one naturally asks whether the QCD description of coherent dijet production can be made fully quantitative.

We developed a perturbative QCD framework for the description of coherent dijet production that is in line with other known applications of

\footnotetext{
*This work was supported by Alexander von Humboldt
} Foundation. the QCD factorization techniques. First we discuss factorization and concentrate on a pion dissociation process, then we will consider production of dijets initiated by a real photon, a process which is sensitive to the chiral-odd properties of QCD vacuum. The results reported here have been obtained in collaboration with V. Braun, S. Gottwald, A. Schäfer and L. Szymanowski [5.6].

\section{Pion dissociation}

The kinematics of the process and the notation for the momenta is shown in Fig. 1. The Sudakov decomposition of the jet momenta with respect to the momenta of the incoming particles $p_{1}$ and $p_{2}$ reads

$q_{1}=z p_{1}+\frac{q_{\perp}^{2}}{z s} p_{2}+q_{\perp}, q_{2}=\bar{z} p_{1}+\frac{q_{\perp}^{2}}{\bar{z} s} p_{2}-q_{\perp}$

so that $z$ is the longitudinal momentum fraction and $q_{\perp}$ the transverse momentum of the quark jet. Hereafter we use the shorthand notation $\bar{u} \equiv$ $(1-u)$ for any longitudinal momentum fraction $u$. Note that we consider the forward limit, when transverse momenta of the jets compensate each other. In this kinematics the invariant mass of the produced $q \bar{q}$ pair is equal to $M^{2}=q_{\perp}^{2} / z \bar{z}$, and the momentum of the outgoing nucleon $p_{2}^{\prime}=p_{2}(1-$ $\xi) /(1+\xi)$, where $\xi=M^{2} /\left(2 s-M^{2}\right) \simeq M^{2} / 2 s$, $s=\left(p_{1}+p_{2}\right)^{2}$.

The possibility to constrain the pion distribution amplitude $\phi_{\pi}(u)$ in the dijet diffractive dissociation experiment assumes that the amplitude 


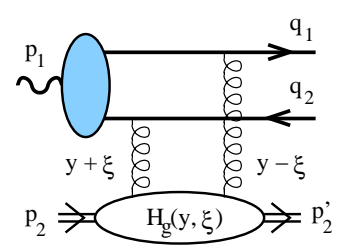

a)

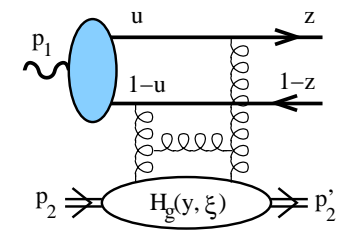

b)
Figure 1. Sample diagrams for the hard dijet production, see text.

of this process can be calculated in the collinear approximation as suggested by Fig. 1:

$M=\int_{0}^{1} d u \int_{-1}^{1} d y \phi_{\pi}(u) T_{H}^{g}(u, y) \mathcal{H}_{g}(y, \xi)$.

Here $\mathcal{H}_{g}(y, \xi)$ is the generalized gluon distribution in the target nucleon [7]; variable $-1<y<$ 1 parametrizes the momentum fractions of the emitted and the absorbed gluons. $T_{H}^{g}(u, y)$ is the hard scattering amplitude involving at least one hard gluon exchange.

There are two important regions in the integral (2), see [5] for more details. At $u \rightarrow 0,1$

$\left.M\right|_{\text {end-points }} \sim i z \bar{z} \int_{u_{\min }}^{1} d u \frac{\phi_{\pi}(u)}{u^{2}} \mathcal{H}_{g}(\xi, \xi)$.

Since $\phi_{\pi}(u) \sim u$ at $u \rightarrow 0$, the integral over $u$ diverges logarithmically. Remarkably, the integral containing the pion distribution amplitude does not involve any $z$-dependence. Therefore, the longitudinal momentum distribution of the jets in the nonfactorizable contribution is calculable and, as it turns out, has the shape of the asymptotic pion distribution amplitude $\phi_{\pi}^{\text {as }}(z)=6 z \bar{z}$.

In technical terms, the appearance of the end point divergence is due to pinching of the $y$ contour at the point $y=\xi$ in case that the variable $u$ is close to the end-points. One can trace [5] that this pinching occurs between soft gluon interactions in the initial and in the final state, and is related with the existence of the unitarity cuts of the amplitude in different, $s$ and $M^{2}$, channels.

The other important integration region in (2) is the one when the longitudinal momentum fraction carried by the quark is close (for high ener- gies) to that of the quark jet in the final state

$$
\left.M\right|_{\xi \ll|u-z| \ll 1} \sim 4 i \phi_{\pi}(z) \int_{\xi}^{1} \frac{d y}{y+\xi} \mathcal{H}_{g}(y, \xi) .
$$

This logarithmic integral is nothing but the usual energy logarithm that accompanies each extra gluon in the gluon ladder. Its appearance is due to the fact the hard gluon which supplies jets by the high transverse momentum can be emitted in a broad rapidity interval and is not constrained to the pion fragmentation region. The integral on the r.h.s. of (1) can be identified with the unintegrated generalized gluon distribution. Therefore, in this region hard gluon exchange can be viewed as a large transverse momentum part of the gluon distribution in the proton, cf. [8]. This contribution is proportional to the pion distribution amplitude $\phi_{\pi}(z)$ and contains the enhancement factor $\ln 1 / \xi \sim \ln s / q_{\perp}^{2}$.

\section{Photon dissociation}

The wave function of a real photon contains both the perturbative chiral-even (CE) contribution of the quark-antiquark pair with opposite helicities, and the nonperturbative chiral-odd (CO) contribution with quarks having the same helicity and which is due to the chiral symmetry breaking. It is proportional to fundamental parameters of QCD vacuum, quark condensate $\langle\bar{q} q\rangle$ and magnetic susceptibility $\chi$. The perturbative CE contribution is singular $\sim 1 /|\mathbf{r}|$ at small transverse distances $\mathbf{r}$. The nonperturbative CO contribution is regular at small transverse separations and can be parametrized by the photon distribution amplitude $\phi_{\gamma}(u, \mu)$ [9]

$$
\begin{aligned}
& \left\langle 0\left|\bar{q}(0) \sigma_{\alpha \beta} q(x)\right| \gamma^{(\lambda)}(q)\right\rangle=i e_{q} \chi\langle\bar{q} q\rangle \times \\
& \left(e_{\alpha}^{(\lambda)} q_{\beta}-e_{\beta}^{(\lambda)} q_{\alpha}\right) \int_{0}^{1} d u e^{-i u(q x)} \phi_{\gamma}(u, \mu) .
\end{aligned}
$$

$\phi_{\gamma}(u, \mu \geq 1 \mathrm{GeV})$ is believed to be not far from the asymptotic form $\phi_{\gamma}^{\text {as }}(u)=6 u(1-u)$. $\chi$ was estimated using the vector dominance approximation and QCD sum rules [10,11: $\chi\langle\bar{q} q\rangle \simeq$ $40-70 \mathrm{MeV}$. However, any direct experimental evidence on both $\chi$ and $\phi_{\gamma}(u)$ is absent. 
This structure can be studied in experiments similar to the studies of coherent dijets in pion dissociation by the E791 collaboration [4]. The

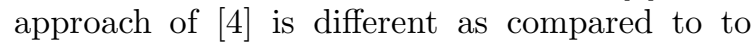
earlier studies of the dijet photoproduction [12] in that the exclusive dijet final state is identified by requiring that the jet transverse momenta are compensated to a high accuracy within the diffractive cone and making some additional cuts. This approach seems to work for the case of pion dissociation, and for photoproduction the corresponding experimental program is under way at HERA 13]. In that case one should take care about the production of heavy quark dijets since for heavy quarks there is a perturbative chiralodd effect due to a quark mass. We assume that separation of the exclusive light $q \bar{q}$ dijet final state is feasible.

Since the CE and CO contributions lead to final states with different helicity, they do not interfere and the dijet cross section is given by the incoherent sum, for the linearly polarized photon

$$
\begin{aligned}
& \left.\frac{d \sigma_{\gamma \rightarrow 2 \text { jets }}}{d \phi d q_{\perp}^{2} d t d z}\right|_{t=0}=\sum_{q} e_{q}^{2} \frac{\alpha_{E M} \alpha_{s}^{2} \pi^{2}(1+\xi)^{2}}{4 \pi N_{c} q_{\perp}^{6}} \times(6) \\
& {\left[\left(1-4 z \bar{z} \cos ^{2} \phi\right)\left|\mathcal{J}_{C E}\right|^{2}+\frac{\pi^{2} \alpha_{s}^{2} \chi^{2}\langle\bar{q} q\rangle^{2}}{N_{c}^{2} q_{\perp}^{2}}\left|\mathcal{J}_{C O}\right|^{2}\right],}
\end{aligned}
$$

where $\phi$ is the azimuthal angle between the jet direction and the photon polarization $\left(e^{(\lambda)} \cdot q_{\perp}\right) \sim$ $\cos \phi, \mathcal{J}_{C E}$ and $\mathcal{J}_{C O}$ are the $\mathrm{CE}$ and $\mathrm{CO}$ amplitudes respectively. Note that the $\mathrm{CE}$ contribution is $\sim 1 / q_{\perp}^{6}$ [3] and the CO contribution is suppressed by one extra power of $q_{\perp}^{2}$ which follows from twist counting. The different $\phi$ dependence can be traced to the fact that the $q \bar{q}$ pair is produced in a state with orbital angular momentum $L_{z}=0$ and $L_{z}= \pm 1$ for the $\mathrm{CO}$ and CE contributions, respectively.

The CE contribution originates from the region of large momenta flowing through the photon vertex and was considered previously in 14, 15, 16, 17 in the high energy limit using $k_{\perp}$ factorization. We believe that the collinear factorization is more adequate for HERA energies an in difference to the dijet production by incident pions, expect that it is valid for the $\mathrm{CE}$ amplitude to all orders in perturbation theory. To leading order $(\mathrm{LO})$ in the strong coupling $\alpha_{s}=\alpha_{s}\left(q_{\perp}\right)$ the amplitude is given by the sum of Feynman diagrams of the type shown in Fig. 1 1 a with all possible attachments of the gluons. The answer reads

$\mathcal{J}_{C E}=i \xi \mathcal{H}_{g}^{\prime}(\xi, \xi)+\frac{i \alpha_{s} N_{c}}{\pi} \int_{\xi}^{1} \frac{d y}{y+\xi} \mathcal{H}_{g}(y, \xi)$,

where $\mathcal{H}_{g}^{\prime}(\xi, \xi)=d \mathcal{H}_{g}(y, \xi) /\left.d y\right|_{y=\xi}$. The second term in eq. (7) originates from the diagrams with additional gluon exchange between the $t$-channel gluons, see Fig. 1 b it corresponds to the leading at large energies (enhanced by $\log \xi$ ) NLO contribution. At $y \rightarrow 0, \mathcal{H}_{g}(y, \xi) \sim y^{-\Delta}$, where in perturbation theory $\Delta \sim \alpha_{s} \log s / q_{\perp}^{2}$ has to be treated as a small parameter. Therefore, despite the fact that the two terms in (7) appear in different orders in the collinear expansion, they are of the same order as far as the counting of energy logarithms is concerned. Since $\mathcal{H}_{g}(y, \xi) \sim G(y)$ at $y \gg \xi$, and as the factor $\alpha_{s} N_{c} /(\pi y)$ is nothing but the low- $y$ limit of the DGLAP gluon splitting function, the integral in Eq. (7) can be identified to logarithmic accuracy with the unintegrated gluon distribution $f\left(\xi, q^{2}\right)=\partial G\left(\xi, q^{2}\right) / \partial \ln q^{2}$. This contribution corresponds one considered in 14, 15,16] in the $k_{\perp}$ factorization approach The first contribution in (7) is analogous to Eq. (42) in 17 .

For the nonperturbative $\mathrm{CO}$ contribution the large momenta are not allowed in the photon vertex and the factorization formula contains a convolution with the photon distribution amplitude. In this case an additional hard gluon exchange is mandatory and the diagram in Fig. 1 $1 \mathrm{~b}$ presents one example of the existing $31 \mathrm{LO}$ contributions. Similar to the pion case we found that the result for the amplitude may be approximated well by the sum of two contributions in analogy to eqs. (3) and (4). The origin of the end-point divergence is the same as in a pion dissociation. In the present context violation of factorization is probably not surprising since the $\mathrm{CO}$ contribution is suppressed by a power of $q_{\perp}^{2}$ compared to the leading twist. Assuming that the photon distribution amplitude is close to the asymptotic form, we obtain $\mathcal{J}_{C O} \sim z(1-z)$ for both integration regions, up to small corrections. The presence of nonfactorizable contribution does not have, there- 
fore, any significant effect on the jet distribution but mainly influences the normalization.

In the numerical calculation performed for HERA kinematics we have introduced an infrared cutoff $u_{\min }=\mu_{\mathrm{IR}}^{2} / q_{\perp}^{2}$ to regularize the nonfactorizable contribution (3), $\mu_{\mathrm{IR}}=500 \mathrm{MeV}$. We found that the nonperturbative $\mathrm{CO}$ contribution integrated over $\phi, z$ and $t$ is of the order of

$\frac{d \sigma_{C O}}{d \sigma_{C E}} \simeq(7 \pm 2 \mathrm{GeV})^{2} \cdot \frac{\alpha_{s}\left(q_{\perp}\right)^{2}}{q_{\perp}^{2}}\left(\frac{\chi\langle\bar{q} q\rangle}{50 \mathrm{MeV}}\right)^{2}$

For $q_{\perp}>4 \mathrm{GeV}$ the cross section is dominated by the perturbative $\mathrm{CE}$ contribution, for smaller transverse momenta the dijet cross section is saturated by the $\mathrm{CO}$ contribution. The transition between the two different regimes is seen very clearly from the dependence of the cross section on the dijet longitudinal momentum fraction a the azimuthal angle. At $q_{\perp}>4 \mathrm{GeV}$ the $z$ distribution is almost flat, while the $\phi$ distribution is almost purely $\sim 1-\cos ^{2} \phi$. In contrast to this at $q_{\perp}<4 \mathrm{GeV}$ the $z$-distribution is comparable with $\sim z^{2}(1-z)^{2}$ while the $\phi$-distribution becomes flat.

One can conclude that studies of exclusive photoproduction of light quark dijets with large transverse momenta can yield important information on the photon structure at small distances. Our main result is that the nonperturbative $\mathrm{CO}$ contribution is large in the region of intermediate $q_{\perp} \sim 2-4 \mathrm{GeV}$ and can be clearly separated from the perturbative contribution by a different $z$ - and $\phi$-dependence. Observation of the CO contribution would be the first clear evidence for the chirality violation in hard processes and also provide the first direct measurement of the magnetic susceptibility of the quark condensate. On the other hand, the dijet cross section for large $q_{\perp}$ can serve to constrain the generalized gluon distribution.

On the theoretical side, we deviate from previous studies of the dijet production by consistently applying the collinear factorization in terms of generalized parton distributions. For the nonperturbative $\mathrm{CO}$ contribution the collinear factorization is, strictly speaking, broken. However, the sensitivity to the infrared cutoff is relatively weak and can formally be eliminated by taking into ac- count Sudakov-type corrections in the modified collinear factorization framework.

\section{REFERENCES}

[1] S.F. King, A. Donnachie and J. Randa, Nucl. Phys. B167 98 (1980);

J. Randa, Phys. Rev. D22 1583 (1980).

[2] G. Bertsch, S.J. Brodsky, A.S. Goldhaber and J.F. Gunion, Phys. Rev. Lett. 47297 (1981).

[3] L. Frankfurt, G.A. Miller and M. Strikman, Phys. Lett. B 3041 (1993).

[4] E.M. Aitala et al. [E791 Collaboration], Phys. Rev. Lett. 864768 (2001); Phys. Rev. Lett. 86 4773 (2001).

[5] V.M. Braun, D.Yu. Ivanov, A. Schäfer and L. Szymanowski, Phys. Lett. B509 43 (2001); Nucl. Phys. B 638 (2002) 111; D.Yu. Ivanov and L. Szymanowski, Phys. Rev. D 64 (2001) 097506.

[6] V.M. Braun, S. Gottwald, D.Yu. Ivanov, A. Schafer and L. Szymanowski, arXiv:hep$\mathrm{ph} / 0206305$.

[7] X.D. Ji, Phys. Rev. D 55, 7114 (1997); A.V. Radyushkin, Phys. Rev. D56 5524 (1997).

[8] N.N. Nikolaev, W. Schäfer and G. Schwiete, Phys. Rev. D63 014020 (2001).

[9] I.I. Balitsky, V.M. Braun and A.V. Kolesnichenko, Nucl. Phys. B 312, 509 (1989).

[10] V.M. Belyaev and Y.I. Kogan, Yad. Fiz. 40 (1984) 1035; I.I. Balitsky, A.V. Kolesnichenko and A.V. Yung, Yad. Fiz. 41 (1985) 282.

[11]P. Ball, V. M. Braun and N. Kivel, hep$\mathrm{ph} / 0207307$.

[12] C. Adloff et al. [H1 Collaboration], Eur. Phys. J. C 6, 421 (1999).

[13] D. Ashery, Nucl. Phys. Proc. Suppl. 108, 321 (2002).

[14] N. N. Nikolaev and B. G. Zakharov, Phys. Lett. B 332, 177 (1994).

[15] J. Bartels, H. Lotter and M. Wusthoff, Phys. Lett. B 379, 239 (1996) [Erratum-ibid. B 382, 449 (1996)].

[16] E. M. Levin, A. D. Martin, M. G. Ryskin and T. Teubner, Z. Phys. C 74, 671 (1997).

[17]K. Golec-Biernat, J. Kwiecinski and A. D. Martin, Phys. Rev. D 58, 094001 
(1998). 